\title{
Detonation wave solutions and linear stability in a four component gas with bimolecular chemical reaction
}

\author{
F. Carvalho, A. W. Silva and A. J. Soares
}

\begin{abstract}
We consider a four component gas undergoing a bimolecular chemical reaction of type $A_{1}+A_{2} \rightleftharpoons A_{3}+A_{4}$, described by the Boltzmann equation (BE) for chemically reactive mixtures. We adopt hard-spheres elastic cross sections and modified line-of-centers reactive cross sections depending on both the activation energy and geometry of the reactive collisions. Then we consider the hydrodynamic limit specified by the reactive Euler equations, in an earlier stage of the chemical reaction, when the gas is far from equilibrium (slow chemical reaction). In particular, the rate of the chemical reaction obtained in this limit shows an explicit dependence on the reaction heat and on the activation energy. Starting from this kinetic setting, we study the dynamics of planar detonation waves for the considered reactive gas and characterize the structure of the steady detonation solution. Then, the problem of the hydrodynamic linear stability of the detonation solution is treated, investigating the response of the steady solution to small rear boundary perturbations. A numerical shooting technique is used to determine the unstable modes in a pertinent parametric space for the considered problem. Numerical simulations are performed for the Hydrogen-Oxygen system and some representative results are presented, regarding the steady detonation wave solution and linear stability.
\end{abstract}

F. Carvalho

Centro de Matemática, Universidade do Minho \& Instituto Politécnico de Viana do Castelo, Portugal e-mail: filipecarvalhodesce.ipvc.pt

A. W. Silva

Instituto Federal do Paraná, Brazil e-mail: adriano.silva@ifpr.edu.br

A. J. Soares

Centro de Matemática, Universidade do Minho, Portugal e-mail: a jsoares@math. uminho. pt 


\section{Introduction}

In a recent paper, Carvalho and Soares [1] consider a model for a two component reacting gas mixture in the framework of the Boltzmann equation and develop a detailed analysis of the dynamics and linear stability of steady detonation wave. This analysis refers to a theoretical detonating mixture of constituents $A$ and $B$ undergoing a generic reversible reaction of type $A+A \rightleftharpoons B+B$. The mathematical treatment of the linear stability of steady detonation waves developed in [1] is rather satisfactory and the numerical technique proposed there can be viewed as an efficient procedure to study the stability problem. Paper [1] also includes an extensive investigation of the stability problem and the results obtained numerically show a rather good qualitative agreement with other results known in literature. However, paper [1] does not explore the study of concrete detonation examples neither the validation of the proposed numerical procedure with respect to the available experimental data. This can be an interesting improvement of the results presented in paper [1].

On the other hand, there exists an increasing interest in detonation physics, from both the experimental and numerical point of view, due to the related engineering applications, and safety and military issues. Experimental observations and numerical studies $[2,3,4,5]$ indicate that the detonation, especially in gases, tends to be unstable. Therefore, the stability analysis of detonation waves remains an interesting topic that has been quite investigated in recent years due to the computational advances.

Motivated by all these aspects, in the present paper we apply the numerical procedure proposed in paper [1] to a different chemically reactive system and investigate the stability of detonation waves in a concrete explosive Hydrogen-Oxygen system. This kinetic formulation refers to a four component gas and adopts a more realistic model of reactive cross sections which modifies the standard line-of-centers model by introducing the dependence on the geometry of the reactive collision. Then we study the dynamics and hydrodynamic linear stability of steady detonation wave solutions, described by the reactive Euler equations obtained in the hydrodynamic limit proper of the initial stage of the chemical reaction.

Numerical simulations are performed for the Hydrogen-Oxygen system and some representative results are presented, regarding the steady detonation wave solution and linear stability.

The present paper constitutes the first part of a work in progress [6] and the numerical results presented here are still limited. We intend to develop a more detailed numerical analysis of the stability problem and, at the same time, to compare our results with other numerical and experimental results available in literature. We expect that such comparisons can be used to reinforce the validity of the numerical procedure presented in paper [1] and consolidate the robustness of the kinetic model proposed in paper [7]. 


\section{The reactive system modelling}

The model adopted in this paper for the reacting gaseous mixture is the one proposed in paper [7] for a four component mixture of constituents $A_{1}, A_{2}, A_{3}$ and $A_{4}$ undergoing the reversible bimolecular chemical reaction

$$
A_{1}+A_{2} \rightleftharpoons A_{3}+A_{4}
$$

Here we include the principal features of the model, with more emphasis on those aspects necessary for our analysis. For a detailed description of the model, see paper [7] and also paper [8] for the foundational aspects of the theory.

\subsection{Modelling aspects}

The constituents of the gas have molecular masses $m_{1}, m_{2}, m_{3}$ and $m_{4}$, molecular diameters $d_{1}, d_{2}, d_{3}$ and $d_{4}$, and binding energies $E_{1}, E_{2}, E_{3}$ and $E_{4}$, respectively. The heat of the chemical reaction is specified by the balance of the binding energies as $Q_{R}=E_{3}+E_{4}-E_{1}-E_{2}$. Molecular masses are such that $m_{1}+m_{2}=m_{3}+m_{4}$, as prescribed by the chemical law. The molecules collide among themselves through binary elastic scattering, and reactive encounters according to the chemical law (1). For elastic scattering, the differential cross sections $\sigma_{\alpha \beta}$ are assumed to correspond to a hard-sphere potential,

$$
\sigma_{\alpha \beta}=d_{\alpha \beta}^{2}, \quad \text { with } \quad d_{\alpha \beta}=\frac{1}{2}\left(d_{\alpha}+d_{\beta}\right)
$$

For reactive encounters, the differential cross sections are assumed with activation energy and dependent on the geometry of the collision, given by

$$
\begin{aligned}
& \sigma_{12}^{\star}= \begin{cases}0 & \text { for } \gamma_{12}<\varepsilon_{f}^{\star}, \\
\mathbf{s}_{f} d_{12}^{2}\left[1-\frac{2 \varepsilon_{f}}{\mu_{12}\left(g_{12} \cdot \mathbf{k}_{12}\right)^{2}}\right] & \text { for } \gamma_{12} \geq \varepsilon_{f}^{\star},\end{cases} \\
& \sigma_{34}^{\star}= \begin{cases}0 & \text { for } \gamma_{34}<\varepsilon_{r}^{\star}, \\
\mathbf{s}_{r} d_{34}^{2}\left[1-\frac{2 \varepsilon_{r}}{\mu_{34}\left(g_{34} \cdot \mathbf{k}_{34}\right)^{2}}\right] & \text { for } \gamma_{34} \geq \varepsilon_{r}^{\star},\end{cases}
\end{aligned}
$$

where $\mu_{12}$ and $\mu_{34}$ are reduced masses, $\gamma_{12}$ and $\gamma_{34}$ relative translational energies in the direction of the line joining the centers of the colliding molecules, $\varepsilon_{f}$ and $\varepsilon_{r}$ forward and reverse activation energies, $\mathbf{s}_{f}$ and $\mathbf{s}_{r}$ are the corresponding steric factors, $\mathbf{k}_{12}$ and $\mathbf{k}_{34}$ unit collision vectors joining the centers of the two colliding molecules pointing from the center of the $A_{2}$ and $A_{4}$-particle to the center of $A_{1}$ and $A_{3}$-particle. Moreover, $g_{12}$ is the pre-collisional asymptotic relative velocity of the constituents $A_{1}$ and $A_{2}$, and $g_{34}$ is the pre-collisional asymptotic relative velocity of $A_{3}$ and $A_{4}$. These kinetic parameters are given by 


$$
\begin{array}{rlrl}
\mu_{12} & =\frac{m_{1} m_{2}}{m_{1}+m_{2}}, & \mu_{34} & =\frac{m_{3} m_{4}}{m_{3}+m_{4}}, \\
\gamma_{12} & =\frac{\mu_{12}\left(g_{12} \cdot \mathbf{k}_{12}\right)^{2}}{2 k T}, & \gamma_{34} & =\frac{\mu_{34}\left(g_{34} \cdot \mathbf{k}_{34}\right)^{2}}{2 k T}, \\
\varepsilon_{f}^{\star} & =\frac{\varepsilon_{f}}{k T}, \quad \varepsilon_{r}^{\star}=\frac{\varepsilon_{r}}{k T}, & Q_{R}^{\star}=\frac{Q_{R}}{k T} \quad \text { with } \quad \varepsilon_{r}^{\star} \equiv \varepsilon_{f}^{\star}-Q_{R}^{\star},
\end{array}
$$

where $k$ represents the Boltzmann constant and $T$ the mixture temperature. Definitions (3) and (4) mean that a reactive collision occurs only when the relative translational energy in the direction of the line joining the centers of the molecules is larger than the activation energy.

Assuming that relativistic and quantum effects are absent, elastic collisions obey the classical laws of mechanics. Therefore, elastic collisions between $A_{\alpha}$ and $A_{\beta}$ molecules, with asymptotic pre-collisional velocities $\mathbf{c}_{\alpha}$ and $\mathbf{c}_{\beta}$ and asymptotic postcollisional velocities $\mathbf{c}_{\alpha}^{\prime}$ and $\mathbf{c}_{\beta}^{\prime}$, respect the following conservation laws of linear momentum and total energy,

$$
\begin{aligned}
& m_{\alpha} \mathbf{c}_{\alpha}+m_{\beta} \mathbf{c}_{\beta}=m_{\alpha} \mathbf{c}_{\alpha}^{\prime}+m_{\beta} \mathbf{c}_{\beta}^{\prime}, \\
& \frac{1}{2} m_{\alpha} \mathbf{c}_{\alpha}^{2}+\frac{1}{2} m_{\beta} \mathbf{c}_{\beta}^{2}=\frac{1}{2} m_{\alpha} \mathbf{c}_{\alpha}^{2}+\frac{1}{2} m_{\beta} \mathbf{c}_{\beta}^{\prime 2} .
\end{aligned}
$$

Furthermore, reactive collisions respect the following conservation laws of linear momentum and total energy (kinetic plus chemical link energy)

$$
\begin{gathered}
m_{1} \mathbf{c}_{1}+m_{2} \mathbf{c}_{2}=m_{3} \mathbf{c}_{3}+m_{4} \mathbf{c}_{4} \\
\frac{1}{2} m_{1} \mathbf{c}_{1}^{2}+\frac{1}{2} m_{2} \mathbf{c}_{2}^{2}=\frac{1}{2} m_{3} \mathbf{c}_{3}^{2}+\frac{1}{2} m_{4} \mathbf{c}_{4}^{2}+Q_{R} .
\end{gathered}
$$

\subsection{The model equations}

The state of a reacting gaseous mixture in the phase space (spanned by the positions $\mathbf{x}$ and velocities $\mathbf{c}_{\alpha}$ ) is characterized, at the mesoscopic level, by the set of distribution functions $f_{\alpha} \equiv f\left(\mathbf{x}, \mathbf{c}_{\alpha}, t\right)$, with $\alpha=1, \ldots, 4$, in such a way that the number of molecules of the contituent $A_{\alpha}$ in the volume element $d \mathbf{x} d \mathbf{c}_{\alpha}$ around the position $\mathbf{x}$ and velocity $\mathbf{c}_{\alpha}$, at time $t$, is given by $f_{\alpha} d \mathbf{x} d \mathbf{c}_{\alpha}$.

The reactive Boltzmann equation that describes the phase space evolution of the distribution functions $f_{\alpha}$, if we consider no external forces and neglect internal degrees of freedom, is given by

$$
\frac{\partial f_{\alpha}}{\partial t}+\sum_{i=1}^{3} c_{i}^{\alpha} \frac{\partial f_{\alpha}}{\partial x_{i}}=\mathscr{Q}_{\alpha}^{E}+\mathscr{Q}_{\alpha}^{R}
$$


Above, $\mathscr{Q}_{\alpha}^{E}$ and $\mathscr{Q}_{\alpha}^{R}$ represent the elastic and the reactive collision terms, respectively, and might be defined as follows

$$
\begin{aligned}
& \mathscr{Q}_{\alpha}^{E}=\sum_{\beta=1}^{4} \mathscr{Q}_{\alpha \beta}^{E}, \quad \text { with } \mathscr{Q}_{\alpha \beta}^{E}=\int\left(f_{\alpha}^{\prime} f_{\beta}^{\prime}-f_{\alpha} f_{\beta}\right) d_{\alpha \beta}^{2}\left(g_{\beta \alpha} \cdot \mathbf{k}_{\beta \alpha}\right) d \mathbf{k}_{\beta \alpha} d \mathbf{c}_{\beta}, \\
& \mathscr{Q}_{1(2)}^{R}=\int\left[f_{3} f_{4}\left(\frac{m_{1} m_{2}}{m_{3} m_{4}}\right)^{3}-f_{1} f_{2}\right] \sigma_{12}^{\star}\left(g_{12} \cdot \mathbf{k}_{12}\right) d \mathbf{k}_{12} d \mathbf{c}_{2(1)}, \\
& \mathscr{Q}_{3(4)}^{R}=\int\left[f_{1} f_{2}\left(\frac{m_{3} m_{4}}{m_{1} m_{2}}\right)^{3}-f_{3} f_{4}\right] \sigma_{34}^{\star}\left(g_{34} \cdot \mathbf{k}_{34}\right) d \mathbf{k}_{34} d \mathbf{c}_{4(3)} .
\end{aligned}
$$

The elastic terms $\mathscr{Q}_{\alpha}^{E}$ incorporate the mixture effects whereas the reactive terms $\mathscr{Q}_{\alpha}^{R}$ include all other effects associated to the chemical reaction, in particular a redistribution of mass and transfer of energy.

\subsection{The consistency of the model}

Some properties are very important in order to show the mathematical and physical consistency of the model. One of these properties states that elastic collisions do not modify the number of molecules of each constituent. This result is ensured by the following statement about the elastic terms defined in (10),

$$
\int_{\mathbb{R}^{3}} \mathscr{Q}_{\alpha}^{E} d c_{\alpha}=0, \quad \alpha=1,2,3,4 .
$$

On the other hand, the reactive encounters imply that the variation of the number of molecules of constituents $A_{1}$ and $A_{2}$ is the same and, at the same time, it is opposite to the variation of the number of molecules of constituents $A_{3}$ and $A_{4}$. This result is stated by the following property on the reactive terms defined in (11) and (12),

$$
\int_{\mathbb{R}^{3}} \mathscr{Q}_{1}^{R} d c_{1}=\int_{\mathbb{R}^{3}} \mathscr{Q}_{2}^{R} d c_{2}=-\int_{\mathbb{R}^{3}} \mathscr{Q}_{3}^{R} d c_{3}=-\int_{\mathbb{R}^{3}} \mathscr{Q}_{4}^{R} d c_{4} .
$$

There are some known physical collisional invariants, that is, macroscopic quantities that do not chance during an elastic collision or reactive encounter. As a consequence, a good and consistent model must reflect this situation. From the mathematical point of view, a function $\psi=\left(\psi_{1}, \psi_{2}, \psi_{3}, \psi_{4}\right)$ is a collisional invariant for the considered model (9-12) if the following conditions hold

$$
\sum_{\alpha=1}^{4} \int_{\mathbb{R}^{3}} \psi_{\alpha}\left(\mathscr{Q}_{\alpha}^{E}+\mathscr{Q}_{\alpha}^{R}\right) d c_{\alpha}=0
$$


The present modelling ensures the conservation of the partial number densities of certain pairs of constituents, namely one reactant and one product. This is a consequence of properties (13) and (14) and reproduces the correct balance of chemical exchange rates. The corresponding collision invariants can be chosen as suitable functions $\psi=\left(\psi_{1}, \psi_{2}, \psi_{3}, \psi_{4}\right)$, defined by $\left(\psi_{1}, \psi_{2}, \psi_{3}, \psi_{4}\right)=(1,-1,0,0)$, $\left(\psi_{1}, \psi_{2}, \psi_{3}, \psi_{4}\right)=(0,1,1,0),\left(\psi_{1}, \psi_{2}, \psi_{3}, \psi_{4}\right)=(0,1,0,1)$. Moreover, the molecular conservation laws (5-8) imply the conservation of the linear momentum components and total energy of the mixture. The corresponding collision invariants can be assumed as functions $\psi=\left(\psi_{1}, \psi_{2}, \psi_{3}, \psi_{4}\right)$ such that $\psi_{\alpha}=m_{\alpha} c_{1}^{\alpha}, \psi_{\alpha}=m_{\alpha} c_{2}^{\alpha}$, $\psi_{\alpha}=m_{\alpha} c_{3}^{\alpha}$, for the linear momentum components, and $\psi_{\alpha}=E_{\alpha}+\frac{1}{2} c_{\alpha}^{2} m_{\alpha}$ for the total energy. In the present model, the set of all collisonal invariants constitute a 7-dimensional linear space.

The consistency of the model based on the properties stated in this subsection allow to derive the macroscopic picture of the kinetic modelling in terms of certain macroscopic variables and balance equations specifying the evolution of such variables. In particular, the macroscopic variables are defined as average quantities taken over the distribution functions $f_{\alpha}$ by integrating with respect to the velocities $\mathbf{c}_{\alpha}$ (see paper [7] for the definitions). The balance equations, in turn, are obtained as a set of seven conservation equations associated to the collisional invariants introduced above, together with the rate equation of the model specifying the evolution of the progress variable of the chemical reaction.

For sake of brevity, these equations are omitted here in their general formulation. For the analysis developed in the present paper, it is enough to consider the onedimensional version of these equations, formulated in its hydrodynamic limit of Euler level, and this will be the main subject of Section 3.

\subsection{Thermodynamical equilibrium}

The reactive mixture is in thermodynamical equilibrium when the elastic and reactive collisional terms are such that

$$
\mathscr{Q}_{\alpha}^{E}+\mathscr{Q}_{\alpha}^{R}=0, \quad \alpha=1, \ldots, 4 .
$$

In particular, for the present model, condition (16) implies the vanishing of the elastic collisional terms, that is

$$
\mathscr{Q}_{\alpha}^{E}=0, \quad \alpha=1, \ldots, 4,
$$

and therefore condition (17) defines a state known in literature as a state of mechanical equilibrium. When all constituents are at the same temperature $T$, the mixture reaches a state of mechanical equilibrium if and only if the distribution functions $f_{\alpha}$ are Maxwellians, defined by 


$$
f_{\alpha}^{M}=n_{\alpha}\left(\frac{m_{\alpha}}{2 \pi k T}\right)^{\frac{3}{2}} \exp \left[-\frac{m_{\alpha}\left(\mathbf{c}_{\alpha}-\mathbf{v}\right)^{2}}{2 k T}\right], \quad \alpha=1, \ldots, 4,
$$

where $n_{\alpha}$ is the number density of constituent $A_{\alpha}$, and $\mathbf{v}$ is the mean velocity of the whole mixture, (see paper [7] for the definitions). The above Maxwellians (18) do not ensure, in general, the vanishing of the reactive collisional operators and thus do not define a state of thermodynamical equilibrium for the reactive mixture. The only distribution function that ensures the thermodynamical equilibrium is the thermodynamical Maxwellian distribution given by

$$
M_{\alpha}=n_{\alpha}^{\mathrm{eq}}\left(\frac{m_{\alpha}}{2 \pi k T}\right)^{\frac{3}{2}} \exp \left[-\frac{m_{\alpha}\left(\mathbf{c}_{\alpha}-\mathbf{v}\right)^{2}}{2 k T}\right], \quad \alpha=1, \ldots, 4,
$$

where $n_{\alpha}^{\mathrm{eq}}$, for $\alpha=1, \ldots, 4$, represent number densities constrained to the law of mass action for the considered model, namely

$$
\ln \left[\frac{n_{1}^{\mathrm{eq}} n_{2}^{\mathrm{eq}}}{n_{3}^{\mathrm{eq}} n_{4}^{\mathrm{eq}}}\left(\frac{\mu_{34}}{\mu_{12}}\right)^{\frac{3}{2}}\right]=Q_{R}^{\star}
$$

which represents the chemical equilibrium condition of the model. Distribution functions (19) define the unique equilibrium solutions of Eqs. (9).

\section{The reactive Euler equations in the hydrodynamic limit}

The reactive Euler equations of the model can be derived from the Boltzmann equations (9), when an approximate solution of Eqs. (9) has been obtained for a prescribed chemical regime.

\subsection{Approximate solution of the Boltzmann equation}

In the present study, we assume that the chemical reaction is in its initial stage corresponding to consider a slow reaction for which the gas mixture is far from chemical equilibrium. In this regime, the elastic collisions are more frequent than reactive encounters. Using the Chapman-Enskog methodology, which is rather common in kinetic theory [9], it is possible to obtain an approximate solution of Eqs. (9) consistent with the prescribed chemical regime.

In paper [7], starting from the appropriate scaling of the Eqs. (9), the ChapmanEnskog methodology has been combined with second-order Sonine expansions of the distribution functions $f_{\alpha}$ around the Maxwellians $f_{\alpha}^{M}$ defined in Eq. (18), and the authors obtained the following approximate solution, 


$$
f_{\alpha}=f_{\alpha}^{M}\left[1+a_{\alpha}\left(\frac{15}{8}-\frac{5 m_{\alpha}\left(c_{\alpha}-v\right)^{2}}{4 k T}+\frac{m_{\alpha}^{2}\left(c_{\alpha}-v\right)^{4}}{8 k^{2} T^{2}}\right)\right], \quad \alpha=1,2,3,4,
$$

where the coefficients $a_{\alpha}$ are determined by the following equations

$$
\begin{aligned}
-\frac{\left(1-e^{-\mathscr{A}^{\star}}\right) \mathbf{s}_{f} d_{12}^{2} e^{-\varepsilon_{f}^{\star}}\left[1-4 \varepsilon_{f}^{\star}+3 \varepsilon_{f}^{\star} E\left(\varepsilon_{f}^{\star}\right) e^{\varepsilon_{f}^{\star}}\right] \mu_{12}^{3 / 2} n_{1} n_{2}}{8 m_{\alpha}^{2}} \\
=\sum_{\beta=1}^{4} \frac{n_{\alpha} n_{\beta} \sqrt{\mu_{\alpha \beta}}}{\left(m_{\alpha}+m_{\beta}\right)^{2}} d_{\alpha \beta}^{2}\left\{\frac{10 m_{\alpha}^{2}+8 m_{\alpha} m_{\beta}+13 m_{\beta}^{2}}{m_{\alpha}+m_{\beta}} a_{\alpha}-15 \mu_{\alpha \beta} a_{\beta}\right\} \\
\quad \text { for } \quad \alpha=1,2, \\
\left\{1-4 \varepsilon_{f}^{\star}+3 \varepsilon_{f}^{\star} E\left(\varepsilon_{f}^{\star}\right) e^{\varepsilon_{f}^{\star}}-4 Q_{R}^{\star}\left[1+Q_{R}^{\star}-\varepsilon_{f}^{\star} E\left(\varepsilon_{f}^{\star}\right) e^{\varepsilon_{f}^{\star}}\left(3-Q_{R}^{\star}\right)\right]\right\} \\
\times \frac{\left(1-e^{-\mathscr{A}}\right) \mathbf{s}_{f} d_{12}^{2} e^{-\varepsilon_{f}^{\star}} \mu_{34}^{5} n_{1} n_{2}}{8 m_{\alpha}^{2} \mu_{12}^{7 / 2}} \\
=\sum_{\beta=1}^{4} \frac{n_{\alpha} n_{\beta} \sqrt{\mu_{\alpha \beta}}}{\left(m_{\alpha}+m_{\beta}\right)^{2}} d_{\alpha \beta}^{2}\left\{\frac{10 m_{\alpha}^{2}+8 m_{\alpha} m_{\beta}+13 m_{\beta}^{2}}{m_{\alpha}+m_{\beta}} a_{\alpha}-15 \mu_{\alpha \beta} a_{\beta}\right\} \\
\quad \text { for } \quad \alpha=3,4 .
\end{aligned}
$$

Above, $\mathscr{A}^{\star}$ is the affinity of the chemical reaction [9], and $E\left(\varepsilon_{f}^{\star}\right)$ represents the exponential integral $E\left(\varepsilon_{f}^{\star}\right)=\int_{\mathcal{E}_{f}^{\star}}^{+\infty} \frac{e^{-y} d y}{y}$. In the present paper, we omit the details of the methodology, they are given in paper [7].

The approximate solution (21) with coefficients specified by expressions (22) and (23) includes the non-equilibrium effects induced by the chemical reaction.

\subsection{Reactive Euler equations}

The reactive Euler equations of the model are obtained from the balance equations, here omitted for sake of brevity, when all macroscopic quantities are expressed in terms of the approximate solution (21-23). They have the form

$$
\begin{aligned}
& \frac{\partial}{\partial t} n_{2}+\sum_{i=1}^{3} \frac{\partial}{\partial x_{i}}\left(n_{2} v_{i}\right)=\tau_{2}, \\
& \frac{\partial}{\partial t}\left(n_{1}-n_{2}\right)+\sum_{i=1}^{3} \frac{\partial}{\partial x_{i}}\left(\left(n_{1}-n_{2}\right) v_{i}\right)=0 \\
& \frac{\partial}{\partial t}\left(n_{2}+n_{3}\right)+\sum_{i=1}^{3} \frac{\partial}{\partial x_{i}}\left(\left(n_{2}+n_{3}\right) v_{i}\right)=0
\end{aligned}
$$




$$
\begin{aligned}
& \frac{\partial}{\partial t}\left(n_{2}+n_{4}\right)+\sum_{i=1}^{3} \frac{\partial}{\partial x_{i}}\left(\left(n_{2}+n_{4}\right) v_{i}\right)=0, \\
& \sum_{i=1}^{3} \frac{\partial}{\partial t}\left(\rho v_{i}\right)+\sum_{i=1}^{3} \sum_{j=1}^{3} \frac{\partial}{\partial x_{j}}\left(p \delta_{i j}+\rho v_{i} v_{j}\right)=0, \\
& \frac{\partial}{\partial t}\left[\frac{3}{2} n k T+\sum_{\alpha=1}^{4} n_{\alpha} E_{\alpha}+\frac{1}{2} \rho v^{2}\right]+\sum_{i=1}^{3} \frac{\partial}{\partial x_{i}}\left[\sum_{j=1}^{3} p \delta_{i j} v_{j}+\right. \\
& \left.\left(\frac{3}{2} n k T+\sum_{\alpha=1}^{4} n_{\alpha} E_{\alpha}+\frac{1}{2} \rho v^{2}\right) v_{i}\right]=0,
\end{aligned}
$$

where $v_{i}, n, \rho$ and $p$ are spatial components of the mean velocity, number density, mass density and pressure of the mixture. The production term $\tau_{2}$ in Eq. (24) is the reaction rate which specifies the progress of the chemical reaction, given by

$$
\tau_{2}=n_{3} n_{4} \tau_{r}-n_{1} n_{2} \tau_{f}
$$

where $\tau_{f}$ and $\tau_{r}$ are forward and backward reaction rates given by

$$
\begin{aligned}
\tau_{f}= & \tau^{(0)}\left\{1-\frac{1-4 \varepsilon_{f}^{\star}+3 \varepsilon_{f}^{\star} E\left(\varepsilon_{f}^{\star}\right) e^{\varepsilon_{f}^{\star}}}{1-\varepsilon_{f}^{\star} E\left(\varepsilon_{f}^{\star}\right) e^{\varepsilon_{f}^{\star}}} \frac{a_{1} m_{2}^{2}+a_{2} m_{1}^{2}}{8\left(m_{1}+m_{2}\right)^{2}}\right\}, \\
\tau_{r}= & \left(\frac{m_{1} m_{2}}{m_{3} m_{4}}\right)^{\frac{3}{2}} e^{Q_{R}^{\star}} \tau^{(0)}\left\{1-\frac{a_{3} m_{4}^{2}+a_{4} m_{3}^{2}}{8\left(m_{3}+m_{4}\right)^{2}}\right. \\
& \left.\times \frac{1-4\left(\varepsilon_{f}^{\star}+Q_{R}^{\star}+Q_{R}^{\star 2}\right)+\varepsilon_{f}^{\star} E\left(\varepsilon_{f}^{\star}\right) e^{\varepsilon_{f}^{\star}}\left(3+12 Q_{R}^{\star}+4 Q_{R}^{\star 2}\right)}{1-\varepsilon_{f}^{\star} E\left(\varepsilon_{f}^{\star}\right) e^{\varepsilon_{f}^{\star}}}\right\},
\end{aligned}
$$

with $\tau^{(0)}$ being the forward reaction rate coefficient given by

$$
\tau^{(0)}=\sqrt{\frac{8 \pi k T}{\mu_{12}}} \mathbf{s}_{f}^{2} d_{12}^{2} e^{-\varepsilon_{f}^{\star}}\left(1-\varepsilon_{f}^{\star} E\left(\varepsilon_{f}^{\star}\right) e^{\varepsilon_{f}^{\star}}\right) .
$$

Equations (24-29) are the reactive Euler equations of the considered model in the adopted chemical regime. They define a closed system and constitute the governing equations of the model. Formally, such equations are similar to the corresponding ones obtained from a phenomenological theory in fluid dynamics. The interesting feature of such equations is that the reaction rate $\tau_{2}$ has been constructed from a kinetic approach and then has an explicit representation completely justified by the microscopic kinetic model. 


\section{Steady detonation wave solutions}

In this section we use the model of Sections 2 and 3 to study the problem of the propagation of steady detonation waves in an explosive quaternary mixture, following the qualitative description of the Zeldovich, von Neumann and Doering (ZND) theory $[2,3]$.

\subsection{The ZND model of detonation}

The well known ZND theory proposes a very simple physical model of detonation with finite chemical reaction zone. The ZND configuration of the detonation solu-

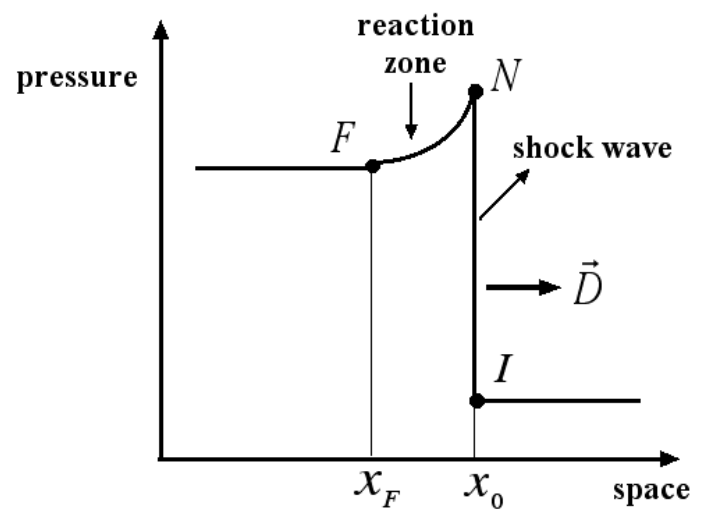

Fig. 1 ZND profile of a one dimentional steady detonation wave.

tion is represented in Figure 1. The solution consists of a strong planar, non-reactive, shock front propagating with constant velocity $D$, greater or equal to its minimum allowed value which is called the Chapman-Jouguet velocity, towards a quiescent gas mixture ahead of the wave. The shock front compresses the mixture, renders the pressure to very high values so that the ignition process takes place. An exothermic chemical reaction initiates and takes place in the finite reaction zone following the shock wave until the equilibrium is reached. The initial state of the quiescent mixture, ahead of the shock wave, is denoted by $I$. The von Neumann state, just ahead of the shock, represents the state with very high pressure where the chemical reaction initiates. The chemical reaction proceeds in the reaction zone of finite length attached to the shock front, until the equilibrium final state denoted by $F$. The entire ZND configuration is steady with respect to the shock wave front.

The ZND theory gives a simplified but recognized description of a steady detonation wave. It is commonly used in literature as the first step in understanding and explaining the complex dynamics of real detonations in gases. 


\subsection{The mathematical approach}

From the mathematical point of view, the ZND detonation solution is described by the reactive Euler equations (24-29), formulated in the one dimensional form and referred to the steady normalized frame attached to the shock wave. The governing equations for the detonation problem become

$$
\begin{aligned}
& \frac{d}{d x}\left[(v-D) n_{2}\right]=D t_{c} \tau_{2}, \\
& \frac{d}{d x}\left[(v-D)\left(n_{1}-n_{2}\right)\right]=0, \\
& \frac{d}{d x}\left[(v-D)\left(n_{2}+n_{3}\right)\right]=0, \\
& \frac{d}{d x}\left[(v-D)\left(n_{2}+n_{4}\right)\right]=0, \\
& \frac{d}{d x}[(v-D) \rho v+n k T]=0, \\
& \frac{d}{d x}\left[(v-D)\left(\frac{3}{2} n k T+\frac{\rho v^{2}}{2}+\sum_{\alpha=1}^{4} E_{\alpha} n_{\alpha}\right)+n k T v\right]=0,
\end{aligned}
$$

where $D$ is the constant shock wave velocity, $x_{s}=\frac{x-D t}{D t_{c}}$ the normalized steady variable, $t_{c}=\frac{1}{4 n^{+} d_{12}^{2}} \sqrt{\frac{M}{\pi k T^{+}}}$a characteristic time, where $M=m_{1}+m_{2}$ is the total mass of the reactants and the superscript + refers to the initial state $I$. For sake of simplicity, the steady variable $x_{s}$ will still be denoted by the plain symbol $x$.

The steady detonation wave problem is solved in two different steps. In the first step, we solve the shock problem to characterize the von Neumann state. This is a pure algebraic problem associated to the Rankine-Hugoniot (RH) jump conditions, and no chemistry is involved. In the second step, we characterize all states within the reaction zone. This is a differential problem associated to the rate law of the chemical reaction and the chemistry plays a relevant role.

Von Neumann state. Since no chemistry is involved in the shock problem, the rate equation (34) becomes of conservative type. The integration of the resulting system (34-39) between the initial state $I$ and the von Neumann state $N$ leads to the RH jump conditions in the form

$$
\begin{aligned}
& n_{\alpha}(v-D)=-n_{\alpha}^{+} D, \quad \alpha=1,2,3,4, \\
& \rho v(v-D)+n k T=n^{+} k T^{+}, \\
& \left(\frac{3}{2} n k T+\frac{\rho v^{2}}{2}+\sum_{\alpha=1}^{4} E_{\alpha} n_{\alpha}\right)(v-D)+n k T v \\
& =-\left(\frac{3}{2} n^{+} k T^{+}+\sum_{\alpha=1}^{4} E_{\alpha} n_{\alpha}^{+}\right) D .
\end{aligned}
$$


For each value of the shock velocity $D$, the RH conditions (40-42) characterize the von Neumann state $\left(n_{1}, n_{2}, n_{3}, n_{4}, v, T\right)$ behind the shock wave, when the initial state $\left(n_{1}^{+}, n_{2}^{+}, n_{3}^{+}, n_{4}^{+}, 0, T^{+}\right)$is assigned.

States in the reaction zone. The intermediate states within the reaction zone describe sequential states of the chemical process and are characterized by integrating the rate equation (34) with initial conditions at the von Neumann state. Using the RH conditions (40) for $\alpha=1,3,4$ together with (41) and (42), we can write the rate equation in the form

$$
\frac{d}{d x} n_{2}=\frac{D t_{c} \tau_{2}}{v-D+n_{2} \frac{d v}{d n_{2}}}
$$

For each value of the shock velocity $D$, and starting from the von Neumann state characterized in the previous step, Eq. (43) together with RH conditions (40) for $\alpha=1,3,4$ as well as (41) and (42) completely characterizes all states in the reaction zone. In particular, the final state of chemical equilibrium is obtained when the reaction rate $\tau_{2}$ vanishes.

The mathematical approach and the solution procedure just described allow to obtain the reaction zone profiles for pressure, mean velocity, temperature, mass density and also the calculation of the wave thickness and other relevant properties in the detonation mechanism.

\subsection{Numerical results for detonation waves in the $\mathrm{H}_{2}-\mathrm{O}_{2}$ system}

In this section we perform some numerical simulations for one dimensional steady detonation waves propagating in the hydrogen-oxygen mixture. We are particularly interested in the elementary chemical reaction

$$
\mathrm{OH}+\mathrm{H}_{2} \rightleftharpoons \mathrm{H}+\mathrm{H}_{2} \mathrm{O}
$$

that is involved in the realistic multi-step detonation mechanism of the hydrogenoxygen mixture.

The initial state of the fresh quiescent mixture and the reference input data for the reaction heat $Q_{R}$ and forward activation energy $\varepsilon_{f}$ are chosen as follows

$$
\begin{aligned}
& n_{\mathrm{OH}}=0.1 \mathrm{~mol} / \mathrm{l}, n_{\mathrm{H}_{2}}=0.2 \mathrm{~mol} / \mathrm{l}, n_{\mathrm{H}}=0.03 \mathrm{~mol} / \mathrm{l}, n_{\mathrm{H}_{2} \mathrm{O}}=0.02 \mathrm{~mol} / \mathrm{l}, \\
& v=0 \mathrm{~ms}^{-1}, T=298.15 \mathrm{~K}, \\
& Q_{R}=-63.3 \mathrm{~kJ} / \mathrm{mol}, \varepsilon_{f}=13.8 \mathrm{~kJ} / \mathrm{mol} .
\end{aligned}
$$

Since $Q_{R}<0$, the forward chemical reaction is exothermic. Our representation of the detonation wave structure is determined using the mathematical modelling described in Subsections 4.1 and 4.2. We obtain some detonation profiles for different values of the detonation wave velocity, namely 


$$
D=3120 \mathrm{~ms}^{-1}, D=3130 \mathrm{~ms}^{-1}, D=4400 \mathrm{~ms}^{-1}, D=4500 \mathrm{~ms}^{-1} .
$$

Figures 2, 3 and 4 show the reaction zone profiles for pressure, temperature and mean velocity, when $D=3120 \mathrm{~ms}^{-1}$ and $D=3130 \mathrm{~ms}^{-1}$. Figures 5, 6 and 7 show the corresponding profiles when $D=4400 \mathrm{~ms}^{-1}$ and $D=4500 \mathrm{~ms}^{-1}$. These figures reproduce the typical ZND configuration for the diagrammed macroscopic variables. In particular, the pictures reveal that, as expected, the width of the reaction zone, that is the wave thickness, decreases with increasing values of the detonation velocity $D$.

To be more precise, the wave thickness is the spatial distance from the shock front to the equilibrium final state, reached when the reaction rate $\tau_{2}$ vanishes and $n_{2}$ becomes constant, see Eq. (43). Thus, considering that the mixture reaches the chemical equilibrium when $\frac{d}{d x} n_{2}<10^{-6}$, the wave thickness can be determined for the detonation velocities defined in (46). The results are given in Table 1.

\begin{tabular}{|c||l|l|l|l|}
\hline$D$ & $3120 m s^{-1}$ & $3130 m s^{-1}$ & $4400 m s^{-1}$ & $4500 m s^{-1}$ \\
\hline Wave thickness & 1.340 & 1.327 & 0.156 & 0.142 \\
\hline
\end{tabular}

Table 1 Wave thickness for different values of the detonation velocity $D$.

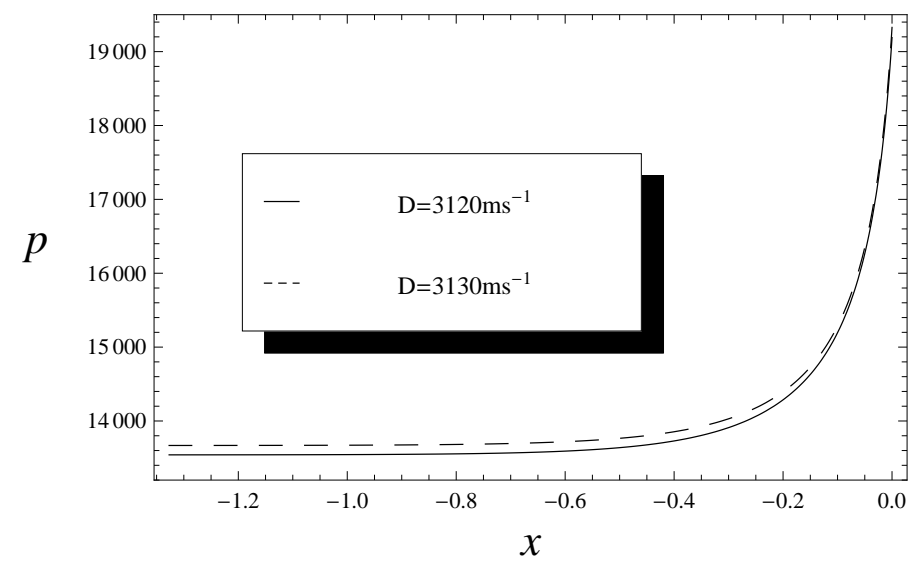

Fig. 2 Pressure profile in the reaction zone for two different wave velocities, $D=3120 \mathrm{~ms}^{-1}$ (solid line) and $D=3130 \mathrm{~ms}^{-1}$ (dashed line). 


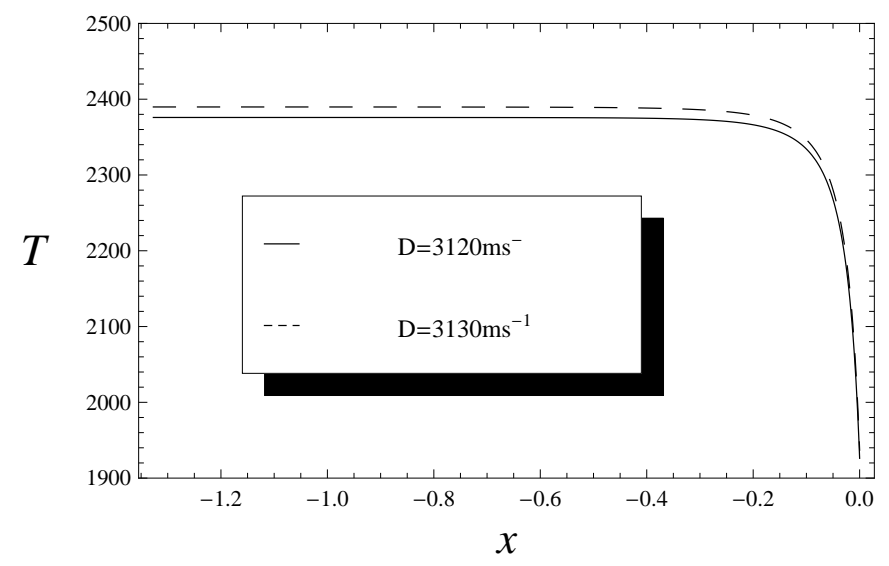

Fig. 3 Temperature profile in the reaction zone for two different wave velocities, $D=3120 \mathrm{~ms}^{-1}$ (solid line) and $D=3130 \mathrm{~ms}^{-1}$ (dashed line).

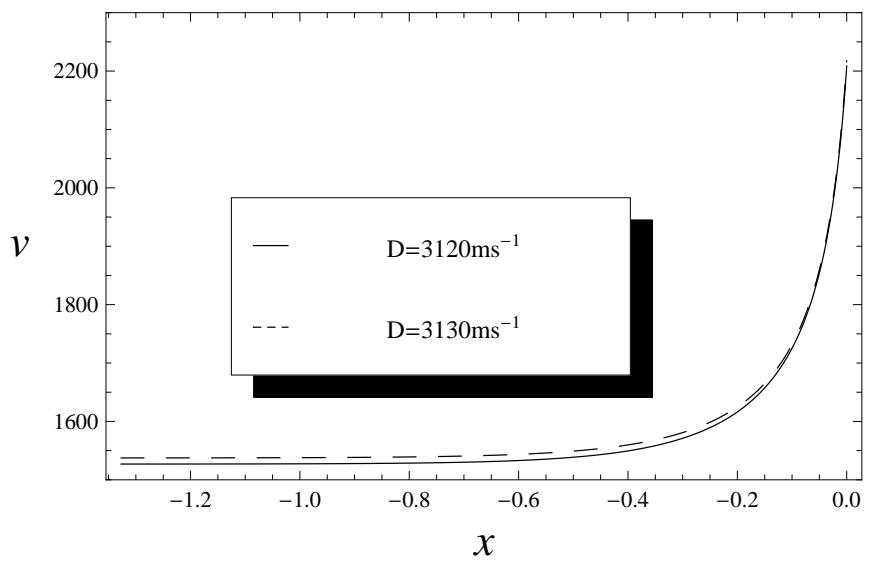

Fig. 4 Mean velocity profile in the reaction zone for two different wave velocities, $D=3120 \mathrm{~ms}^{-1}$ (solid line) and $D=3130 \mathrm{~ms}^{-1}$ (dashed line).

\section{Linear stability analysis}

Experimental and numerical studies show that detonations tend to be structurally unstable, particularly in gases, see [2] and [3]. The reaction zone is extremely sensitive to small perturbations and the detonation wave typically exhibits oscillating instabilities, which become more pronounced when the shock front propagates with velocity close to its minimum value. The evolution of such instabilities and a systematic analysis about the unstable modes, neutral stability boundaries and growth rates of the instabilities can be of crucial importance in the interpretation of the complex detonation mechanism. From the mathematical point of view, this stabil- 


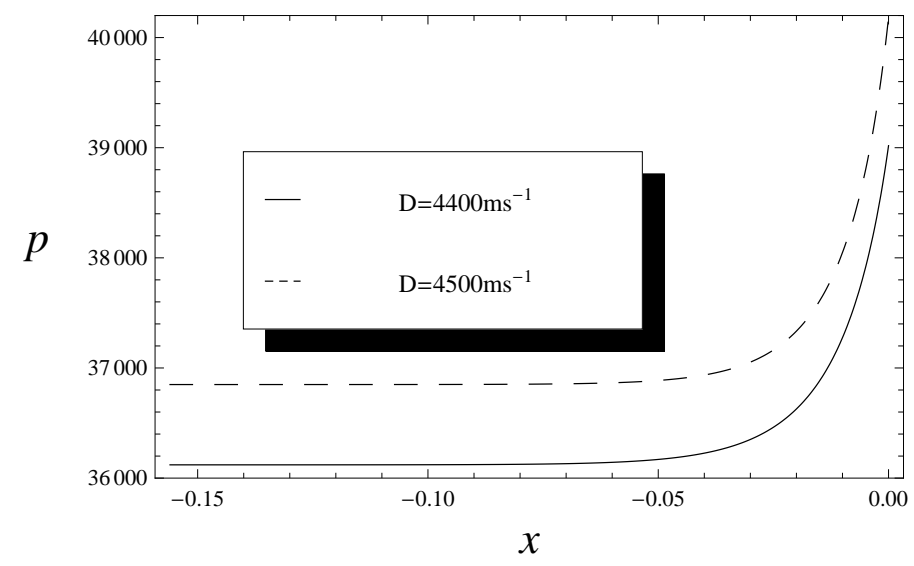

Fig. 5 Pressure profile in the reaction zone for two different wave velocities, $D=4400 \mathrm{~ms}^{-1}$ (solid line) and $D=4500 \mathrm{~ms}^{-1}$ (dashed line).

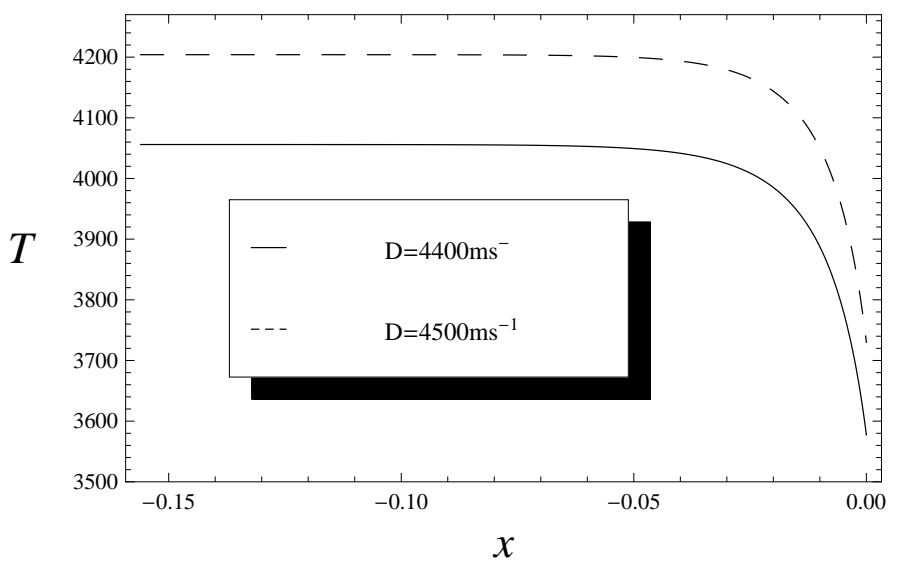

Fig. 6 Temperature profile in the reaction zone for two different wave velocities, $D=4400 \mathrm{~ms}^{-1}$ (solid line) and $D=4500 \mathrm{~ms}^{-1}$ (dashed line).

ity analysis can be developed using a normal-mode linear approach of the steady planar detonation solution. This linear approach is valid when one investigates the effects induced by small perturbations and assumes that the steady structure of the detonation wave is not significantly modified.

In this section we formulate the linear stability problem for the steady detonation solution characterized in Section 4, then we describe the numerical technique used in the simulations and, finally, we present some results about the detonation instability. 


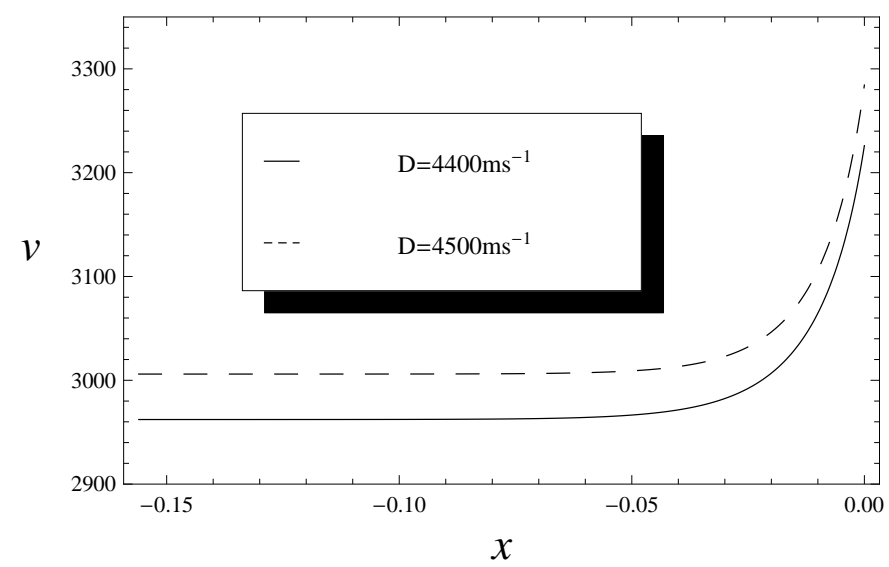

Fig. 7 Mean velocity profile in the reaction zone for two different wave velocities, $D=4400 \mathrm{~ms}^{-1}$ (solid line) and $D=4500 \mathrm{~ms}^{-1}$ (dashed line).

\subsection{Stability problem}

The problem is formulated assuming that a small perturbation is instantaneously assigned at the rear boundary and a distortion on the shock wave position occurs. As a result, the shock distortion affects the steady character of the reaction zone and the objective is to investigate the dynamics of the perturbations induced on the macroscopic variables representing the steady detonation wave in the reaction zone.

First, the one-dimensional closed governing equations (34-39) are transformed to the coordinate frame attached to the perturbed wave. A new wave coordinate is introduced, $x$, which measures the distance from the perturbed shock,

$$
x=x^{\ell}-\psi(t), \quad \text { with } \quad \psi(t)=D t+\widetilde{\psi}(t),
$$

where $x^{\ell}$ is the laboratory coordinate, $\psi(t)$ the position of the perturbed wave in the laboratory frame, and $\widetilde{\psi}(t)$ the spatial displacement of the perturbed shock with respect to its unperturbed position. The shock position in the new frame is $x=0$ and the shock velocity is $D(t)=D+\widetilde{\psi}^{\prime}(t)$. Then, to describe the oscillatory behaviour of the instabilities, we perform a normal mode expansion of the steady state variables and perturbed shock position, in the form

$$
z(x, t)=z^{*}(x)+e^{a t} \bar{z}(x) \quad \text { and } \quad \psi(t)=\bar{\psi} e^{a t}, \quad \text { with } \quad a, \bar{\psi} \in \mathbb{C},
$$

where we have used the vectorial notation for the state fields, $z=\left[\begin{array}{lllll}n_{1} & n_{2} & n_{3} & n_{4} & v\end{array}\right]^{T}$. Here, $z^{*}(x)$ represents the state vector of the steady solution, $\bar{z}(x)$ the vector of the spatial disturbances of the steady state fields, $\bar{\psi}$ the disturbance amplitude parameter, and $a$ is a perturbation parameter such that $\operatorname{Re} a$ and $\operatorname{Im} a$ are the perturbation growth rate and frequency, respectively. 
We linearize Eqs. (34-39) by means of expansions (48) and normalize the state variables with respect to the complex amplitude parameter $\bar{\psi}$. For sake of simplicity, we keep the original notation $\bar{z}$ for the normalized variables. The resulting equations constitute the stability equations of the present problem and have the form

$$
\begin{aligned}
& D a \bar{n}_{2}+\left(v^{*}-D\right) \frac{d}{d x} \bar{n}_{2}+(\bar{v}-a) \frac{d}{d x} n_{2}^{*}+\bar{n}_{2} \frac{d}{d x} v^{*}+n_{2}^{*} \frac{d}{d x} \bar{v}=\bar{\tau}_{2}, \\
& D a\left(\bar{n}_{1}-\bar{n}_{2}\right)+\left(v^{*}-D\right) \frac{d}{d x}\left(\bar{n}_{1}-\bar{n}_{2}\right)+(\bar{v}-a) \frac{d}{d x}\left(n_{1}^{*}-n_{2}^{*}\right) \\
& +\left(\bar{n}_{1}-\bar{n}_{2}\right) \frac{d}{d x} v^{*}+\left(n_{1}^{*}-n_{2}^{*}\right) \frac{d}{d x} \bar{v}=0, \\
& D a\left(\bar{n}_{2}+\bar{n}_{3}\right)+\left(v^{*}-D\right) \frac{d}{d x}\left(\bar{n}_{2}+\bar{n}_{3}\right)+(\bar{v}-a) \frac{d}{d x}\left(n_{2}^{*}+n_{3}^{*}\right) \\
& +\left(\bar{n}_{2}+\bar{n}_{3}\right) \frac{d}{d x} v^{*}+\left(n_{2}^{*}+n_{3}^{*}\right) \frac{d}{d x} \bar{v}=0, \\
& D a\left(\bar{n}_{2}+\bar{n}_{4}\right)+\left(v^{*}-D\right) \frac{d}{d x}\left(\bar{n}_{2}+\bar{n}_{4}\right)+(\bar{v}-a) \frac{d}{d x}\left(n_{2}^{*}+n_{4}^{*}\right) \\
& +\left(\bar{n}_{2}+\bar{n}_{4}\right) \frac{d}{d x} v^{*}+\left(n_{2}^{*}+n_{4}^{*}\right) \frac{d}{d x} \bar{v}=0, \\
& \rho^{*} D t_{c} a \bar{v}+\frac{d}{d x} \bar{p}+\rho^{*}(\bar{v}-a) \frac{d}{d x} v^{*}+\left(\bar{\rho} \frac{d}{d x} v^{*}+\rho^{*} \frac{d}{d x} \bar{v}\right)\left(v^{*}-D\right)=0, \\
& D t_{c} a \bar{p}+\frac{5}{3}\left(p^{*} \frac{d}{d x} \bar{v}+\bar{p} \frac{d}{d x} v^{*}\right)+\left(v^{*}-D\right) \frac{d}{d x} \bar{p}+(\bar{v}-a) \frac{d}{d x} p^{*}=\frac{2 Q_{R}^{\star} \bar{\tau}_{2}}{3},
\end{aligned}
$$

where $\bar{\tau}_{2}$ is the linearized representation of the reaction rate, given by

$$
\bar{\tau}_{2}=\left(n_{3}^{*} \bar{n}_{4}+n_{4}^{*} \bar{n}_{3}\right) \tau_{r}^{*}+\left(n_{1}^{*} \bar{n}_{2}+n_{2}^{*} \bar{n}_{1}\right) \tau_{f}^{*} .
$$

We also linearize the Rankine-Hugoniot conditions (40-42) using the normal mode expansions (48), obtaining

$$
\begin{aligned}
& \bar{n}_{\alpha}(0)=\frac{\left(n_{\alpha}^{*}-n_{\alpha}^{+}\right) a-n_{\alpha}^{*} \bar{v}(0)}{v^{*}-D}, \quad \alpha=1,2,3,4, \\
& \bar{v}(0)=\frac{3 \rho^{+} v^{* 2}+\frac{3}{2}\left(p^{*}-p^{+}\right)-\frac{3}{2} D \rho^{+} v^{*}+\sum_{\alpha=1}^{4} E_{\alpha} n_{\alpha}}{-\rho^{*}\left(v^{*}-D\right)^{2}+\frac{5}{2} p^{*}} a, \\
& \bar{p}(0)=-\rho^{+} a v^{*}-\left(v^{*}-D\right) \rho^{*} \bar{v}(0) .
\end{aligned}
$$

From the linearization procedure, we obtain twelve real first-order homogeneous ODE's, Eqs. (49-54), to be considered in the reaction zone from $x=0$ at the von Neumann state to $x=x_{F}$ at the equilibrium final state, with twelve real initial conditions given by Eqs. (56-58). The equations involve twelve unknowns specified by the real and imaginary parts of $\bar{n}_{1}, \bar{n}_{2}, \bar{n}_{3}, \bar{n}_{4}, \bar{v}, \bar{p}$. Since the equations involve the complex perturbation parameter $a$, the ODE system is not closed, and an additional closure condition is needed. We assign the following boundary condition, at $x=x_{F}$, initially proposed and justified by Buckmaster and Ludford in [10], 


$$
\bar{v}\left(x_{F}\right)+a=\frac{-1}{\gamma \rho_{e q}^{*} c_{e q}^{*}} \bar{p}\left(x_{F}\right),
$$

where $\gamma$ is the ratio of specific heats, and $c_{e q}^{*}$ and $\rho_{e q}^{*}$ are the isentropic sound speed and mixture mass density at $x=x_{F}$.

The stability problem just formulated will be numerically solved as described in the next subsection.

\subsection{Numerical technique}

The numerical technique used in this paper to treat the stability problem is based on an iterative shooting algorithm proposed in Ref. [1]. The algorithm combines the numerical approach developed by Lee and Stewart in paper [11] with the original ideas advanced by Erpenbeck in paper [12].

Broadly speaking, the technique consists in choosing, first, a trial value for the perturbation parameter $a$, then solving the ODE's (49-54) with initial conditions (5658 ) and, finally, verifying if the solution previously obtained satisfies the boundary condition (59). If the boundary condition is satisfied, the corresponding solution of (49-54) and (56-58) represents a stability solution, that is a solution of the stability problem.

After finding a solution of the stability problem, for a trial value of $a$, the last step is straightforward. In fact, it only requires to determine if the solution of the stability problem produces a stable or an unstable mode of propagation, and this is as follows: If $\operatorname{Re} a>0$, then the parameter $a$ results in an unstable mode; if $\operatorname{Re} a<0$, then it results in a stable mode.

The conclusion of the stability analysis is the following. The steady detonation solution is stable when all solutions of the stability problem result in stable modes of propagation. Conversely, it is unstable when at least one solution of the stability problem results in an unstable mode.

The main numerical difficulty of the stability analysis is to find solutions of the stability problem. In fact, an arbitrary trial value of $a$ does not satisfy, in general, the boundary condition (59). This difficulty has been solved by Carvalho and Soares in paper [1], using a numerical technique that combines ideas and methodologies of previous works, as mentioned above.

Accordingly, we introduce the residual function $\mathscr{H}$ in a fixed domain $\mathscr{R} \subset \mathbb{C}$,

$$
\mathscr{H}(a)=\bar{v}\left(x_{F}\right)+a+\frac{1}{\gamma \rho_{e q}^{*} c_{e q}^{*}} \bar{p}\left(x_{F}\right), \quad a \in \mathscr{R} \subset \mathbb{C},
$$

and notice that the zeros of $\mathscr{H}$ satisfy the boundary condition (59). Thus, resorting to the argument principle, first used by Erpenbeck in [12], and taking into account that the function $\mathscr{H}$ has no poles in $\mathscr{R}$, we count the number $Z$ of zeros of $\mathscr{H}$ by means of the expression 


$$
Z=\frac{1}{2 \pi i} \int_{k}^{\ell} \frac{\mathscr{H}^{\prime}(\zeta(t))}{\mathscr{H}(\zeta(t))}\left\|\zeta^{\prime}(t)\right\| d t
$$

where $\zeta:[k, \ell] \rightarrow \mathbb{C}$ is a path smooth by parts, describing the contour of $\mathscr{R}$ in the positive sense.

Starting from these ideas, the numerical procedure used to solve the stability problem consists in nine steps described below.

1. Choose the domain $\mathscr{R}$ in the complex plane where we intend to look for eigenvalues of the stability problem.

2. Define a path $\zeta$ describing the contour of $\mathscr{R}$ in the positive sense.

3. Select a great number of trial values $a_{j}$ in the contour of $\mathscr{R}$.

4. Introduce further trial values $b_{j}$ defined by $b_{j}=a_{j}+10^{-6}$.

5. Solve the stability governing equations (49-54) with initial conditions (56-58) for each trial value $a_{j}$ and $b_{j}$ for the perturbation parameter $a$.

6. Evaluate the residual function $\mathscr{H}$ at each point $a_{j}$ and $b_{j}$.

7. Estimate the derivative $\mathscr{H}^{\prime}\left(a_{j}\right)$ by the quotient $\left(\mathscr{H}\left(b_{j}\right)-\mathscr{H}\left(a_{j}\right)\right) /\left(b_{j}-a_{j}\right)$.

8. Estimate the mean value $\mu$ of the function $\frac{\mathscr{H}^{\prime}(\zeta(t))}{\mathscr{H}(\zeta(t))}\left\|\zeta^{\prime}(t)\right\|$ using a suitable sample and a $99 \%$ confidence interval.

9. Count the number of zeros of the residual function $\mathscr{H}$, approximating the expression on the right hand-side of Eq. (61) by $Z=\frac{1}{2 \pi i}(k-\ell) \mu$.

The details of the numerical procedure and a rather complete discussion on the technique can be found in Ref. [1].

\subsection{Results and discussion}

Using the numerical procedure described in the previous subsection, we were able to obtain some results on the stability behaviour of the steady detonation solution described in Section 4. Considering the reference input data indicated in (45) and the values of the detonation wave velocity $D$ referred in (46), we obtain some estimations for the number of unstable modes in the region $\mathscr{R}$ defined by

$$
0.001<\operatorname{Re} a<0.1 \quad \text { and } \quad 0.001<\operatorname{Im} a<0.1 .
$$

The estimations are presented in Table 2 and represent very preliminary results on the stability behaviour of the steady detonation solution described in Section 4.

The determination of instability solutions is a very complex task and a time consuming problem. In Table 2 we present some estimations for the number of instability modes obtained for different values of the detonation velocity. These results are still rough approximations and should be improved. In fact, it is known that the number of instability modes grows as the detonation velocity approaches its minimum value, see for example Refs. [2] and [11]. The results presented in Table 2 show that unstable modes exist for lower values of the detonation velocity, as it is 


\begin{tabular}{|c|c|}
\hline$D$ & Number of unstable modes \\
\hline \hline $3120 \mathrm{~ms}^{-1}$ & 12 to 334 \\
\hline $3130 \mathrm{~ms}^{-1}$ & 158 to 684 \\
\hline $4400 \mathrm{~ms}^{-1}$ & 0 \\
\hline $4500 \mathrm{~ms}^{-1}$ & 0 \\
\hline
\end{tabular}

Table 2 Estimations for the unstable modes in the region $\mathscr{R}$, for fixed values of the reaction heat $Q_{R}^{*}$ and activation energy $\varepsilon_{f}^{*}$, and for different values of the detonation velocity $D$.

expected from the literature. However, since the confidence intervals obtained for $D=3120 \mathrm{~ms}^{-1}$ and $D=3130 \mathrm{~ms}^{-1}$ show a significant overlap, we are not able to compare the number of instability modes for these two detonation velocities.

The results obtained in the present paper are still very limited and should be improved. This will be addressed in a work in progress [6], where more accurate simulations will be conducted and further detailed results will be included. Among several interesting topics, we intend to investigate the following two issues: the limit detonation velocity characterizing stable solutions for other detonating of Hydrogen-Oxygen mixtures defined in terms of different constituent concentrations; the relation between the detonation wave velocity and the number of instability modes. Moreover other simulations will be performed oriented to compare our results with others available in literature, obtained from numerical studies and experimental works.

Acknowledgements This paper was partially supported by the Research Centre of Mathematics of the University of Minho through Portuguese funds from the "Fundação para a Ciência e a Tecnologia", through the Project PEstOE/MAT/UI0013/2014. The author Silva, A.W. acknowledges the support by the Instituto Federal do Paraná - Campus of Curitiba, Brazil.

\section{References}

1. Carvalho, F., Soares, A.J.: On the dynamics and linear stability of one-dimensional steady detonation waves. J. Phys. A: Math. Theor. 45, 255501, 1-23 (2012)

2. Fickett, W., Davis, W.C.: Detonation, Theory and Experiment. University of California Press, Berkeley (1979)

3. Lee, J.H.S.: The Detonation Phenomenon. Cambridge University Press, Cambridge (2008)

4. Oran, E.S., Boris, J.P.: Numerical Simulation of Reactive Flow. Cambridge University Press, New York (2001)

5. Oran, E.S., Weber Jr, J.W., Stefaniw, E.I., Lefebvre, M.H., Anderson Jr, J.D.: A Numerical Study of a Two-Dimensional H2-O2-Ar Detonation Using a Detailed Chemical Reaction Model. Combust. Flame. 113, 147-163 (1998)

6. Carvalho, F., Silva, A.W., Soares, A.J.: Work in preparation about the dynamics, stability and numerical simulations of the steady detonation waves (2014)

7. Kremer, G.M., Silva, T.G.: Analysis of the reaction rate coefficients for slow bimolecular chemical reactions. Braz. J. Phys.. 42, 400-409 (2012) 
8. Kremer, G.M., Silva, A.W., Alves, G.M.: On inelastic reactive collisions in kinetic theory of chemically reacting gas mixtures. Physica A. 389, 2708-2718 (2010)

9. Kremer, G.M.: Introduction to the Boltzmann Equation and Transport Processes in Gases. Springer, Berlin (2010)

10. Buckmaster, J.D., Ludford G.S.S.: The effect of structure on the stability of detonations I. Role of the induction zone. In: Twenty-First Symposuim (International) on Combustion, pp. 1669-1676. The Combustion Institute. Elsevier (1986)

11. Lee, H.I, Stewart, D.S.: Calculation of linear detonation stability: one dimensional instability of plane detonation. J. Fluid Mech. 216, 103-132 (1990)

12. Erpenbeck, J.J.: Stability of steady-state equilibrium detonations. Phys. Fluids. 5, 604-614 (1962) 